\title{
Desigualdad global, poder y el mundo unipolar: implicancias para la educación en Trabajo Social
}

\section{Global inequality, power and the unipolar world: implications for Social Work education}

\section{PhD. James Midgley}

James Midgley es Harry and Riva Specht Professor, Dean Emeritus, 1997-2006 de la School of Social Welfare en UC Berkeley University.203 Haviland Hall Phone: (510)642-4430; midg@berkeley.edu

\begin{abstract}
Resumen
James Midgley hace un llamado a que la preocupación tradicional del trabajo social respecto de la desigualdad en el ingreso y la riqueza como también de las opresiones étnicas, de género y culturales, sea ampliada hacia un entendimiento de cómo las relaciones globales de poder operan y afectan las condiciones sociales con que los trabajadores sociales primariamente intervienen.

Palabras clave (Trabajo social, mundo unipolar, globalización, poder.)

Abstract

James Midgley appeals to the traditional concern of social work regarding the inequality in income and wealth as well as the oppression of ethnic, gender and cultural rights, asking to extend it toward an understanding of how the overall relations of power operate and affect social conditions in which social workers are primarily involved.
\end{abstract}

Key words (Social work, unipolar world, globalization, power)

La elección de Crecimiento y Desigualdad como tema para el Congreso 2006 IASSW es bienvenida y oportuna. Aunque la desigualdad ha sido un tema destacado en los debates políticos, económicos y sociales durante los siglos XIX y XX, el argumento de que la desigualdad tiene una importancia secundaria para alcanzar la meta de promover un crecimiento económico rápido, ha sido ampliamente aceptado en años recientes. Desde los años 80, con el auge de la ideología capitalista neo-liberal, se ha sostenido exitosamente que el crecimiento más que la redistribución debe ser prioritario en la política económica. Muchos líderes políticos y gestores de políticas han sido convencidos por este argumento. Aún los líderes de partidos políticos como la Social Democracia Progresiva, o como el Partido Liberal Inglés, han aceptado el panorama que la desigualdad es un "no tema".

Sin embargo, durante los últimos años el tema de la desigualdad se ha retomado en la literatura académica y popular. Varios estudios han mostrado que las desigualdades en ingreso y riqueza en muchos países se han extendido y aparte de sus efectos sociales negativos, muchos creen que estas desigualdades tienen consecuencias políticas y económicas dañinas. Organizaciones Internacionales como por ejemplo el Banco Mundial, conceden ahora que el rápido crecimiento económico durante estas dos últimas décadas, no ha traído prosperidad a todos. En muchas partes del mundo, el rápido crecimiento económico ha sido acompañado por un estancamiento de los ingresos de las personas comunes. Por otro lado la riqueza y el crecimiento se concentran cada vez más. Hoy, la necesidad de orientar el problema parece estar reconocido más ampliamente.

Discusiones recientes respecto a la desigualdad se han relacionado primariamente con la disparidad en ingreso y riqueza, manteniendo la antigua práctica establecida de usar indicadores monetarios para medir la desigualdad. Sin embargo, la literatura no 
ha prestado mucha atención a las desigualdades en razas, sexos y las amplias y poderosas relaciones que éstas reflejan, una auténtica y comprensiva conceptualización de la desigualdad debe abarcar estas formas de opresión. Por consiguiente, existe una necesidad urgente de sintetizar los asuntos cuantitativos y economicistas de la academia convencional y profundizar en el tema de la desigualdad, con el insight del multiculturalismo y la sociología, perspectivas que analizan la desigualdad desde una matriz no-monetaria.

El estudio de la desigualdad necesita también fijar su atención en las relaciones de poder internacionales y la forma como diferentes grados de poder entre naciones a nivel global influencian las desigualdades domésticas y locales. A pesar de los ideales cosmopolitas-igualitarios contenidos en las cartas de organizaciones multilaterales como las Naciones Unidas, los países insertos en el sistema mundial moderno no son similares en el sentido de ejercer influencia económica y política. En realidad a los países miembros de las Naciones Unidas y otras organizaciones multilaterales se les recuerda frecuentemente de cómo sus intereses y esfuerzos están sujetos a relaciones globales de poder desiguales. Estas desigualdades tienen una penetrante influencia que afecta un amplio espectro de problemas, incluyendo de forma ostensible asuntos sociales no controversiales, de salud y medio ambiente como por ejemplo el calentamiento global, planificación familiar y prevención del sida.

Es por esta razón que los estudios de desigualdad global deben ser informados a través de una comprensión actualizada en el campo académico de las relaciones internacionales. Mientras que el estudio de relaciones internacionales no ha sido de mucho interés para académicos e investigadores del trabajo social, la preocupación tradicional del trabajo social respecto de la desigualdad en el ingreso y la riqueza como también de las opresiones étnicas, de género y culturales, necesita ser ampliada por un entendimiento de cómo las relaciones globales de poder operan y afectan las condiciones sociales con que los trabajadores sociales primariamente intervienen.

\section{Perspectivas teóricas respecto al poder global}

Una enriquecida herencia de pensamiento académico respecto a de qué manera las diversas personas del mundo debiera interactuar se ha ido acumulando durante siglos. Mucho antes de que emergieran los estados-nación modernos, pensadores políticos y sociales de distintas eras ofrecieron interpretaciones de cómo y por qué el poder es ejercido entre personas de distintas etnias y nacionalidades y cómo personas de distintas culturas y sociedades debieran relacionar entre sí. En el pensamiento social y político de Occidente comentarios normativos al respecto, pueden ser rastreados hasta llegar a las ideas de Cínicos y Estoicos, siendo después reformulados en las teologías de Agustín y Aquinas, quienes enfatizaron los aspectos en común compartidos en la humanidad de todas las personas.

El cosmopolitismo clásico puso la primera piedra para la subsiguiente internacionalización de Kant, Hegel, Marx y Engels, y en tiempos más recientes dio inspiración para los actuales multilateralistas tales como los fundadores de la Liga de las Naciones y las Naciones Unidas. Pero mientras el ideal Kantiano de los estados naciones cooperando entre sí como socios igualitarios bajo la dirección de entidades gubernamentales supranacionales para promover intereses nacionales y globales continúa dotando de una razón poderosa para la cooperación internacional, también tiene muchos detractores.

Por ejemplo, los etnonacionalistas vienen sospechando desde hace tiempo de los motivos de los internacionalistas los cuales, según ellos, intentan absorber la soberanía nacional con un mal definido gobierno mundial a futuro. Rechazan la idea que la ciudadanía derivada de la identidad étnica-nacional deba sumergirse bajo la rúbrica de la "Ciudadanía Global". Aunque no rechazan la cooperación internacional, están comprometidos a mantener la identidad nacional y proteger los intereses nacionales. Por consiguiente, ellos buscarán ventaja nacional al relacionarse con la política internacional y perseguirán políticas diseñadas para mantener la lealtad doméstico-cultural.

Tanto las posiciones nacionalistas y cosmopolitas son rechazadas por isolacionistas, quienes sostienen que los estados naciones están mejor cuando enfocan problemas domésticos y evitan embrollos internacionales. En consecuencia, proponen que el compromiso internacional debe ser minimizado. Su posición es rechazada por aquellos que manifiestan que en el mundo globalizado de hoy, es simplemente imposible evitar "embrollos" internacionales. Les guste o no las naciones derivan inexorablemente hacia los sistemas políticos y económicos globales, viéndose afectados por el comercio, tecnologías comunicacionales, viajes, diplomacia y otros eventos internacionales. 
Este último argumento es reforzado por los realistas internacionales quienes dominan las relaciones internacionales hoy en día. Toman como punto de vista que el idealismo en el pensamiento cosmopolita es digno de admiración, pero simplemente no implementable. Las naciones estados, sostienen, actuarán de manera tal que promocionaran sus propios intereses. El mejor acercamiento es enfrentar estas realidades y esperar que la competencia por el poder -como la competencia económica- sea sana y positiva. Si la competencia por el poder internacional tiene efectos negativos, como el fomentar el militarismo, es probable que "el balance de poder" que asegura la mantención por siempre de la paz, emerja. Mientras los realistas crean que las reglas y regulaciones internacionales sean necesarias para sostener la arquitectura internacional, ellos se darán cuenta que estas reglas serán latamente determinadas por aquellos capaces de ejercer el poder global.

Una variación extrema del acercamiento realista es sustentada por imperialistas unipolares, los cuales creen que los estados poderosos van a, inevitablemente, dominar a las naciones más débiles siendo esta una manera natural y realista de organizar los asuntos internacionales. Sus puntos de vista han sido usados no sólo para justificar el ejercicio de la diplomacia, del poder militar y económico, sino que también justifican la difusión de valores e instituciones. A diferencia de los multilateralistas Kantianos, los imperialistas de hoy en día recurren a ideas Hobbesianas para sostener que el mundo es un lugar extremadamente peligroso compuesto por estados-naciones egoístas y agresivos. Estando por encima de la perspectiva del balance del poder, proponen que las naciones del mundo pongan su confianza en un poderoso pero benevolente Leviathan capaz de usar su poder militar, diplomático y económico para mantener la paz en el mundo. Señalan que en el pasado los imperios Británico y Romano jugaron este rol. Los unipolaristas actuales creen que EE.UU es el único calificado para asumir esta responsabilidad.

Estas ideas normativas no han sido adecuadamente debatidas por los trabajadores sociales interesadas en asuntos internacionales. Si bien la literatura sobre trabajo social internacional se ha visto influenciada por el espíritu Kantiano de cooperación y mutualidad, también ha sido influenciada por puntos de vista menos optimistas respecto a la posibilidad de trabajar la cooperación internacional entre trabajadores sociales en diferentes partes del mundo. Por ejemplo, en los `70 algunos trabajadores sociales en el Sur Global fueron influenciados por el estructuralismo internacional Neo-Marxista y vieron a las relaciones internacionales dentro de lo profesional como "imperialistas". Por otra parte, la teoría post colonial ha ejercido relativamente poca influencia en el pensamiento del trabajo social, aún cuando Midgley (1998) hizo notar que las ideas post-colonialistas podían fortalecer una mejor evaluación de cómo las continuidades coloniales influían las desigualdades a nivel doméstico e internacional. En el nivel doméstico, un análisis de la perpetuación de la pobreza y deprivación entre los indígenas en sociedades de colonos es incisivamente enfatizado por interpretaciones post-coloniales.

La complejidad de estas teorías y los temas a menudo contradictorios que emergen para el trabajo social internacional no han sido debatidos adecuadamente. Por ejemplo, el desafío planteado por el pensamiento post-moderno al cosmopolitanismo inherente al trabajo social internacional no ha sido totalmente examinado. De forma similar, se ha escrito suficiente respecto a la globalización pero, de nuevo, muy pocos autores de trabajo social han reconocido hasta qué punto los elementos cosmopolitas en el discurso de la globalización han tenido implicancias positivas, a fin de fortalecer la cooperación global de la sociedad civil y patrocinar una política social internacional. Estos complejos temas, necesitan ser desenredados y sometidos a continuos debates no sólo para efectos académicos sino que prácticos. Como se hizo notar anteriormente, el compromiso de trabajo social profesional en un sin número de campos prácticos es directamente afectado por relaciones de poder internacional. El entender la complejidad de las teorías e ideologías que dan expresión a estas relaciones de poder y las cuales, a su vez, las influencian, es vital para formular posiciones en muchos asuntos claves del trabajo social.

\section{El mundo unipolar}

Es en este marco que el concepto de unipolarismo toma una particular relevancia respecto al trabajo social internacional. Popularizado por el escritor norteamericano, neoconservador, Charles Krauthammer a principios de los '90 (vea Krauthammer, 2004), el término evoca una nueva imagen global, la cual reemplaza conceptualizaciones y clasificaciones previas de los estados países del mundo. Incluye la dicotomía bi-polar "Este v/s Oeste" de la Guerra Fría y la clasificación tri-polar "Tres Mundos" defendida por los líderes del movimiento no-alineado de 
los '50. Para el movimiento no-alineado el mundo estaba dividido en tres grandes esferas de influencia o "polos"; a saber, las democracias capitalistas occidentales, la Unión Soviética y los países en desarrollo. Formulaciones subsiguientes aumentaron esta clasificación al agregar un "Cuarto Mundo" de países extremadamente pobres en desarrollo o por ver al mundo compuesto por algunas pocas esferas distintas, culturales o "civilizacionales" como aquellas que concibió Samuel Huntisgton (1996) en su libro "El Choque de Civilizaciones". Para los cosmopolitas, sin embargo, el mundo está mejor descrito, no como una multiplicidad de polos, sino que como una comunidad de estados-nación iguales y soberanos unidos por leyes internacionales y participando en una base recíproca de esfuerzos cooperativos globales.

Intelectuales neo conservadores y políticos se mofan ante este concepto, señalando que las relaciones internacionales no se han caracterizado por idealismos, sino que por duras realidades políticas y poder económico. Estas realidades más que idealistas, lo que hacen es reclamar mutuo respeto y reciprocidad. La mirada realista fue promovida activamente por escritores neoconservadores, tales como Normal Podhoretz e Irving Kristal en los `80, los cuales propugnaron que las políticas acomodaticias de la era de la guerra fría fueron rechazadas, y que el gobierno de los EE.UU se abocara totalmente a la destrucción de la Unión Soviética y de su ideología comunista. Su destrucción, sostenían, era necesaria para asegurar la paz mundial y promover el bienestar social y económico de los habitantes del mundo. Despreciaban al Presidente Jimmy Carter y sus esfuerzos de promover la paz a través del diálogo, apoyaban a los ataques de Ronald Reagan hacia la Unión Soviética y manifestaban que la obstinada idea de Reagan fue la que llevó al colapso Soviético en una nueva era de paz y prosperidad global.

Subsiguientemente, una nueva generación de neoconservadores incluyendo a Richard Perle, Paul Wolfowitz, Robert Kogan y William Kristal habían articulado una visión para la diplomacia americana la cual propone que el gobierno de los EE.UU, acepte sus nuevas responsabilidades como la única Superpotencia Mundial y, en el nuevo mundo unipolar, no tolerará ninguna oposición de ningún lado. Los americanos, sostiene, no deben tener reserva alguna para aceptar su nuevo rol como guardianes del orden mundial. De forma interesante esta visión es apoyada por algunos eruditos de otras partes del mundo, como por ejemplo, el notable historiador británico Niall Ferguson y el economista indio Deepack Lal, quienes han avalado de forma entusiasta los ideales de la nueva PAX AMERICANA.

La idea de Krauthammer, del mundo unipolar, encontró eco en el informe de política interna del Departamento de Estado en 1992, el cual requirió el reemplazo de la estrategia de la Guerra Fría de "internacionalismo colectivo", por una nueva estrategia de "dominación benevolente". Esbozada en gran parte por Paul Wolfowitz, bajo la supervisión del Secretario de Defensa Dick Cheney, el informe político requirió a la primera administración de Bush declarar la total hegemonía de los EE.UU y asumir la responsabilidad única por la seguridad internacional. Aunque el informe político recibió una acogida hostil, al filtrarse al New York Times, sus premisas más importantes fueron reiteradas agresivamente durante los ' 90 por numerosos pensadores neoliberales con el apoyo de los centros de investigación (think tanks) del ala gobernante de derecha.

En 1997, un grupo de neoconservadores bajo la dirección de William Kristol y Gary Schmith fundaron el Proyecto para un Nuevo Siglo Americano el cual promueve el uso del poder diplomático, económico y militar para difundir los valores e ideales americanos. Tal como los romanos dieron forma a su mundo, también los líderes del Proyecto propugnaron al gobierno para re-hacer el nuevo mundo ante la imagen americana. En 1998 los líderes neoconservadores enviaron una carta abierta al Presidente Clinton proponiendo una agresiva estrategia político-militar para derrocar la dictadura de Saddam Hussein en Irak. Haciéndose llamar Comité Para La Paz y Seguridad en el Golfo, el grupo incluía luminarias actuales tales como el Secretario de Defensa Donald Rumsfeld, el Presidente del Banco Mundial Paul Wolfowitz, el Embajador de EE.UU ante Las Naciones Unidas John Bolton y el Embajador en Irak Zolmay Khalilzad.

En su reciente libro, El Caso de Goliat, Michael Mandelbaum (2005) ofrece una nueva y audaz interpretación del unipolarismo, argumentando que los debates acerca de las ventajas y desventajas respecto de la hegemonía global americana han sido presentadas dudosamente desde que el gobierno de los EE.UU., ha, de hecho, emergido (y ha sido ampliamente aclamado) como el gobierno de facto del mundo. Las ideas de Mandelbaum dan última 
expresión a los elementos neo-hobbesianos dentro de la ideología unipolar que señalan que la paz mundial puede llegar a ser bajo directrices de un Leviethan poderoso con la voluntad y medios para controlar los estados-nación.

Mientras que la tesis de Mandelbaum puede ser también interpretada cínicamente sugiriendo que el cosmopolismo ideal para el gobierno mundial ahora ya se ha realizado, los cosmopolitas se pueden horrorizar ante la idea de que un poder imperial puede, efectivamente, representar los intereses de las diversas naciones del mundo y personas. El unipolarismo acaba con el ideal Post II Guerra Mundial, como se demuestra en la Carta de las Naciones Unidas, el cual mencionaba que el mundo debe estar compuesto de una comunidad igualitaria de países estados soberanos cooperando entre ellos y viviendo en paz bajo una ley internacional.

Los defensores del unipolarismo no sólo han ridiculizado el ideal cosmopolita, sino que han hecho campañas en su contra. Ejercieron influencias sobre círculos de política exterior norteamericana en los '90, y obviamente, sus ideas fueron adoptadas por la actual administración Bush, la cual frecuentemente ha declarado su compromiso de expandir las ideas de democracia liberal y capitalismo de libre mercado a través del mundo. El ejemplo más dramático de la adopción de este unipolarismo neoconservador fue la invasión a Irak. El desprecio unipolarista hacia las Naciones Unidas se reveló ante la insistencia de que el gobierno de EE.UU tiene el derecho, bajo la doctrina del pre-despojo, de actuar de forma unilateral en la arena internacional. Desde luego los unipolaristas han previamente exhortado al Congreso Norteamericano a rechazar una variedad de tratados internacionales y acuerdos sobre derechos humanos, y a desdeñar organizaciones multilaterales tales como la Corte Criminal. Más recientemente, han asegurado el nombramiento de unipolaristas en puestos claves de instituciones internacionales, como por ejemplo, el Banco Mundial y las Naciones Unidas. Sus ataques también se han dirigido hacia organizaciones internacionales sin fines de lucro que no cuentan con su aprobación en los campos ideológicos. El gobierno de Bush ha usado su considerable poderío económico y diplomático para influenciar sobre la política mundial de medio ambiente, derechos humanos, salud reproductiva y los esfuerzos para prevenir el SIDA, particularmente en África. Por ejemplo, un artículo reciente de la revista norteamericana EL PROSPECTO AMERICANO (The American Project) (Klaplan,
2006) muestra como las tradicionales políticas de la administración Bush han tenido consecuencias negativas para prevenir el SIDA en Uganda donde el soporte financiero para programas de promoción de la abstinencia ha tenido como resultado la disminución del uso de preservativo y el correspondiente incremento e incidencia de las infecciones del VIH.

\section{Sugerencias para la educación en Trabajo Social}

La agenda unipolarista representa un desafío mayor para los docentes de trabajo social y profesionales que creen en los ideales de la cooperación y reciprocidad internacional. Una gran cantidad del esfuerzo internacional del trabajo social se fundamenta en estos ideales. A través de los años los trabajadores sociales han formado asociaciones profesionales a nivel nacional, regional e internacional que promueven la cooperación entre ellos en diferentes partes del mundo. Estas organizaciones y los trabajadores sociales que ellas representan, son respetuosas de las diferencias y buscan comprender y compartir más que imponer puntos de vista profesionales. Cuando estos ideales se confirmen en las reuniones internacionales de este verano, y en futuros eventos internacionales, los trabajadores sociales deben ser cuidadosos respecto al rol de la ideología unipolar y las poderosas fuerzas sociales hegemónicas que se han liberado.

La ideología unipolar tiene profunda implicancia para los trabajadores sociales docentes preocupados de la desigualdad en el mundo. Al impartir enseñanzas sobre desigualdad y opresión, los educadores del trabajo social deben estar atentos sobre la manera de cómo la relación del poder global tiene su efecto sobre las desigualdades domésticas y la vida de las personas, familias y comunidades que ellos sirven. Un conocimiento de las realidades del poder global debe formar parte de las agendas de investigación. Aún más, los educadores del trabajado social deben insistir en que sus asociaciones profesionales, nacionales e internacionales, confronten incidentes específicos que reflejen el brutal desarrollo del poder global y reafirmen de manera práctica su compromiso con el diálogo y entendimiento internacional. Esto implica algo más que declaraciones opositoras a las desigualdades causadas por globalización económica o a opresiones étnicas, religiosas, homofóbicas y otras. La defensa del trabajo social debe ir más allá de exhortar a estudiantes en las salas de clases y con publicaciones del mismo tipo. Requiere 
un compromiso específico para desafiar a aquellos con poder en niveles nacionales e internacionales que buscan minar los esfuerzos de los multilateristas que han luchado por promover la cooperación internacional en la post era de la segunda guerra mundial. Las asociaciones profesionales de asistentes sociales, como tales, son incapaces de ejercer mucha influencia sobre los defensores del unipolarismo. Sin embargo, formando coaliciones con otras organizaciones internacionales progresivas, movimientos y grupos, los trabajadores sociales pueden contribuir en positivas formas a enfrentar el problema de la desigualdad global, promover la paz, los derechos humanos, la justicia social internacional y el bienestar de todos los ciudadanos del mundo.

\section{Referencias}

KAPLAN, E. (2006). "Fairy-Tale Failure." The American Prospect. July/August, 2006, p. 9.

HUNTINGTON, S. P. (1996). The clash of civilizations and the remaking of the world order. New York: Simon \&Schuster.

KRAUTHAMMER, C. (2004). Democratic realism: An American foreign policy for a unipolar world. Washington DC: AEI Press.

MANDELBAUM, M. (2005). The case for goliath: How America acts as the world's government in the 21st century. New York: Public Affairs Press.

MIDGLEY, J. (1998). 'Colonialism and welfare: A PostColonialcCommentary.' Journal of Progressive Human Services. 9 (2), 31-50. 